\title{
Anti-oxidative and Anti-inflammatory Activities of Polysaccharide isolated from Korean-Style Soy Sauce
}

\author{
Hoon Kim ${ }^{1, *}$, Jungeun Park ${ }^{2, * *}$, Jaemee Jung ${ }^{2, * * *}$ and Dahyun Hwang ${ }^{2,3, ; ;, * * * *}$ \\ ${ }^{I}$ Skin-biotechnology Center, Kyung Hee University, Suwon-si, Gyeonggi-do 16229, Korea \\ ${ }^{2}$ Department of Biomedical Laboratory Science, College of Life and Health Sciences, \\ Hoseo University, Asan, Chungnam 31499, Korea \\ ${ }^{3}$ The Research Institute for Basic Sciences, Hoseo University, Asan, Chungnam 31499, Korea
}

Soy sauce is one of the representatives of traditional fermented foods in Korea. However, studies on soy sauce are relatively insufficient in Korea compared to Japan. In this study, antioxidant and anti-inflammatory activities of polysaccharides were measured by polysaccharides isolated from two different soy sauces, Korean and Japanese (KSS-0 and JSS-0). KSS-0 was purified into two fractions using gel chromatography and named them as KSS-I and KSS-II. To investigate the antioxidant activity of the polysaccharides, we measured the polyphenol content and radical scavenging activity using 2,2-diphenyl-1-picrylhydrazyl (DPPH) and 2,2'-azino-bis (3-ethylbenzothiazoline-6-sulfonic acid) (ABTS). To investigate the anti-inflammatory activity of polysaccharides, we used RAW 264.7 macrophage cells and induced inflammation using lipopolysaccharide (LPS). Then, we measured levels of inflammatory mediators such as nitric oxide (NO) and tumor necrosis factor (TNF)- $\alpha$. Among the four polysaccharides, KSS-II showed the highest antioxidant activity and had good anti-inflammatory activity; KSS-II decreased inflammatory mediators in a dose-dependent manner. In conclusion, the polysaccharide isolated from Korean soy sauce (KSS-II) showed better anti-oxidant and anti-inflammatory activities than polysaccharides isolated from Japanese soy sauce, and may be useful as substances for functional foods.

Key Words: Soy sauce, Anti-oxidant, Anti-inflammation, Polysaccharide, Functional foods

Soy sauce is a representative fermented food of Korea, which contains soybean as the main ingredient. As interest in health increases worldwide, interest in fermented foods is also increasing. There are some studies on the functional activities of Japanese soy sauce, such as anti-allergic activity (Kobayashi, 2005) and antiplatelet activity (Tsuchiya et al., 1999). However, studies on Korean soy sauce are relatively insufficient. Japanese and Korean soy sauce have some differences, due to variability in raw materials, fermentation methods, and microbial differentiation. Japanese soy sauce blends soybeans and wheat flour in the 'Koji' method (Uchida et al., 2019). Korean soy sauce is fermented using only soy beans in the 'Mezu' method (Yang et al., 2012). Therefore, the physiological activities of Korean and Japanese soy sauce are expected to be different.

Oxidation is a normal process in living organisms to produce energy. In normal or pathological cell metabolism, free radicals are produced (Shirley et al., 2014). However,

Received: January 29, 2020 / Revised: February 18, 2020 / Accepted: March 6, 2020

${ }^{*}$ Research professor, ${ }^{* *}$ Researcher, ${ }^{* * *}$ Undergraduate student, ${ }^{* * * *}$ Professor.

${ }^{\dagger}$ Corresponding author: Dahyun Hwang. Department of Biomedical Laboratory Science, Hoseo University, 20, Hoseo-ro 79 beon-gil, Baebang-eup, Asan-si, Chungcheongnam-do 31499, Korea.

Tel: +82-41-540-9628, Fax: +82-41-540-9997, e-mail: hdh@hoseo.edu

(C) The Korean Society for Biomedical Laboratory Sciences. All rights reserved.

(c) This is an Open Access article distributed under the terms of the Creative Commons Attribution Non-Commercial License (http://creativecommons.org/licenses/by-nc/3.0/) which permits unrestricted non-commercial use, distribution, and reproduction in any medium, provided the original work is properly cited. 
if control over oxygen-derived free radicals is lost, they produce many diseases, such as cancer, rheumatoid arthritis, and arteriosclerosis (Dore et al., 2007; Shirley et al., 2014). Inflammation is also a normal immune process to protect the host when pathogens invade the body. However, uncontrolled inflammation causes diverse diseases such as rheumatoid arthritis, atherosclerosis, inflammatory bowel disease, and asthma (Tak and Firestein, 2001). Therefore, it is very important to have the proper level of oxidation and inflammation in the body.

The need for safer drugs, without side effects, is increasing. Of alternative sources, polysaccharide has been reported for its multiple biological activities including as an anticarcinogenic, anticoagulant, immuno-stimulant, and antioxidant (Wang et al., 2013). Therefore, in this study, we used polysaccharide isolated from soy sauce and compared the anti-oxidative and anti-inflammatory activities between polysaccharides from Korean and Japanese soy sauce.

In this experiment, Japanese soy sauce was used from the Japanese company ' $\mathrm{K}$ ' and Korean soy sauce was used from the Korean traditional food manufacturer 'S'. To isolate and purify polysaccharide, Korean and Japanese soy sauce was dissolved overnight by adding approximately 4-fold ethanol to $80 \%$ of the total concentration. After the supernatant was separated by centrifugation $(6,000 \mathrm{rpm}, 30 \mathrm{~min})$, it was precipitated with three volumes of ethanol and dialyzed for two days using dialysis tubing (molecular weight cut off [MWCO]: 12,000 14,000; Sigma Aldrich, St. Louis, MO, USA). The solution was finally lyophilized to yield crude polysaccharides named as JSS-0 and KSS-0. To further purify KSS-0, crude polysaccharide was applied to an open $(4 \mathrm{~cm} \times 95 \mathrm{~cm})$ Sephadex G-75 column (GE Healthcare Life Sciences, Uppsala, Sweden), equilibrated with $50 \mathrm{mM}$ ammonium formate buffer ( $\mathrm{pH} 5.5$ ), and eluted with the same buffer. Two major purified polysaccharides (KSS-I and KSS-II) were obtained and lyophilized after desalting by dialysis.

To investigate the anti-oxidative activities, the total polyphenol content and free radical scavenging activities were measured using 2,2'-azino-bis (3-ethylbenzothiazoline-6sulfonic acid) (ABTS) and 2,2-diphenyl-1-picrylhydrazyl (DPPH). The total polyphenol content of polysaccharides was determined using the Folin-Ciocalteu method with some modifications (Singleton and Rossi, 1965). Each extract $(20 \mu \mathrm{L})$ was mixed with $100 \mu \mathrm{L}$ of Folin-Ciocalteu reagent (Sigma chemical Co., St. Louis, MO, USA) and reacted for three min at room temperature (RT) in the dark. Then, the mixture was added to $80 \mu \mathrm{L}$ of $7.5 \% \mathrm{Na}_{2} \mathrm{CO}_{3}$ and placed in the dark for $20 \mathrm{~min}$ at RT. The absorbance was measured at $765 \mathrm{~nm}$ using a SpectraMax microplate reader (Molecular Devices, Sunnyvale, CA, USA). The total polyphenol content was calculated based on a calibration curve obtained with gallic acid, and expressed as gallic acid equivalents per gram of dry weight (mg gallic acid eq./g). The ABTS radical scavenging activity was determined using the method of Arnao et el. with some modifications (Arnao et al., 2001). To make ABTS stock solution, 7.4 mM ABTS solution and $2.6 \mathrm{mM}$ potassium persulfate solution were mixed in equal amounts and allowed to incubate for $12 \mathrm{~h}$ at RT in the dark. Then, the stock solution was diluted to obtain an absorbance of 0.7 at $734 \mathrm{~nm}$ using a spectrophotometer. Polysaccharides $(10 \mu \mathrm{L})$ were allowed to react with one $\mathrm{mL}$ of the ABTS stock solution and the absorbance was measured after $30 \mathrm{~min}$ at $734 \mathrm{~nm}$ using a spectrophotometer. The ABTS scavenging activity of the extracts were compared with that of ascorbic acid and expressed as mg ascorbic acid equivalent antioxidant capacity (mg ascorbic acid eq./g) sample. The DPPH radical scavenging activity was measured using the Blois method with some modifications (Blois, 1958). Polysaccharides $(10 \mu \mathrm{L})$ were allowed to react with $100 \mu \mathrm{L}$ of $0.2 \mathrm{mM}$ DPPH (Sigma chemical Co., St. Louis, MO, USA) in a 96well plate. The mixture was shaken vigorously in the dark at RT for $30 \mathrm{~min}$, then the absorbance was measured at $517 \mathrm{~nm}$. The DPPH radical scavenging activity of the extracts were compared with that of ascorbic acid and expressed as mg ascorbic acid equivalent antioxidant capacity (mg ascorbic acid eq./g) sample. To measure the anti-inflammatory activity of the extract, we used RAW 264.7 macrophage cells and induced inflammation using LPS (Sigma, St. Louis, MO, USA). Dulbecco's modified Eagle's medium (DMEM), and penicillin/streptomycin (P/S) were purchased from Gibco BRL Co. (Grand Island, NY, USA), and fetal bovine serum (FBS) was purchased from Welgene Inc. (Gyeongsan, Korea). A mouse TNF- $\alpha$ enzyme-linked immunosorbent assay 
(ELISA) kit was purchased from BD Biosciences (San Diego, CA, USA).

To measure cell viability, RAW 264.7 cells were cultured in DMEM supplemented with $10 \% \mathrm{FBS}$ and $1 \% \mathrm{P} / \mathrm{S}$ at $37^{\circ} \mathrm{C}$ in a humidified incubator with $5 \% \mathrm{CO}_{2}$. When cells reached $70 \sim 80 \%$ confluence, cells were seeded into plates for the assays. Cell viability was measured using the WSTbased reagent EZ-CyTox (Daeil Lab Service, Seoul, Korea). Pre-confluent RAW 264.7 cells $\left(2 \times 10^{5}\right.$ cells $\left./ \mathrm{mL}\right)$ were seeded into 96-well plates overnight. Subsequently, the cells were treated with polysaccharides for $30 \mathrm{~min}$, and cells were co-treated with LPS $(1 \mu \mathrm{g} / \mathrm{mL})$ for $24 \mathrm{~h}$. The culture medium was removed and $100 \mu \mathrm{L}$ of a 1:10 dilution of EZ-CyTox in PBS was added; cells were then incubated for $1 \mathrm{~h}$ at $37^{\circ} \mathrm{C}$ in an atmosphere of $5 \% \mathrm{CO}_{2} / 95 \%$ air. The absorbance was measured at $450 \mathrm{~nm}$ using a SpectraMax microplate reader. Cell viability was expressed as a percentage relative to the deionized water-treated negative control cells.

To investigate anti-inflammatory effects, we measured the inflammatory mediators, nitric oxide (NO) and tumor necrosis factor $\alpha$ (TNF- $\alpha)$. RAW 264.7 macrophages $(2 \times$ $10^{5}$ cells $/ \mathrm{mL}$ ) were cultured in 96 -well plates overnight.
The cells were treated with polysaccharides and 30 min later, cells were co-treated with LPS $(1 \mu \mathrm{g} / \mathrm{mL})$ for $24 \mathrm{~h}$. Secretion of NO was measured using a microplate assay method as described previously (Kim et al., 1999). To measure nitrite $\left(\mathrm{NO}_{2}{ }^{-}\right), 100 \mu \mathrm{L}$ of culture supernatant was mixed with an equal volume of Griess reagent ( $1 \%$ sulfanilamide $/ 0.1 \% \mathrm{~N}$ [1-naphthyl]-ethylenediamine dihydrochloride $/ 2.5 \% \mathrm{H}_{2} \mathrm{PO}_{4}$ ) and incubated for $10 \mathrm{~min}$ at RT. The nitrite concentration was determined by measuring the absorbance at $540 \mathrm{~nm}$. Sodium nitrite $\left(\mathrm{NaNO}_{2}\right)$ was used as a standard. TNF- $\alpha$ levels were measured using an ELISA kit according to the manufacturer's instructions.

All statistical analyses were performed using SPSS version 12.0 for Windows (SPSS, Chicago, IL, USA). Values are expressed as the mean \pm standard deviation (SD) of three independent experiments, performed in triplicate. $P<0.05$ was considered significant (Student's $t$-test). Statistical differences among groups were evaluated with analysis of variance (ANOVA), followed by Duncan's multiple range tests.

Two crude polysaccharides were isolated from the hot water extract of Japanese soy sauce (JSS) and Korean soy sauce (KSS) by $80 \%$ ethanol precipitation and named JSS-0 and KSS- 0 . KSS-0 showed higher antioxidant activity than

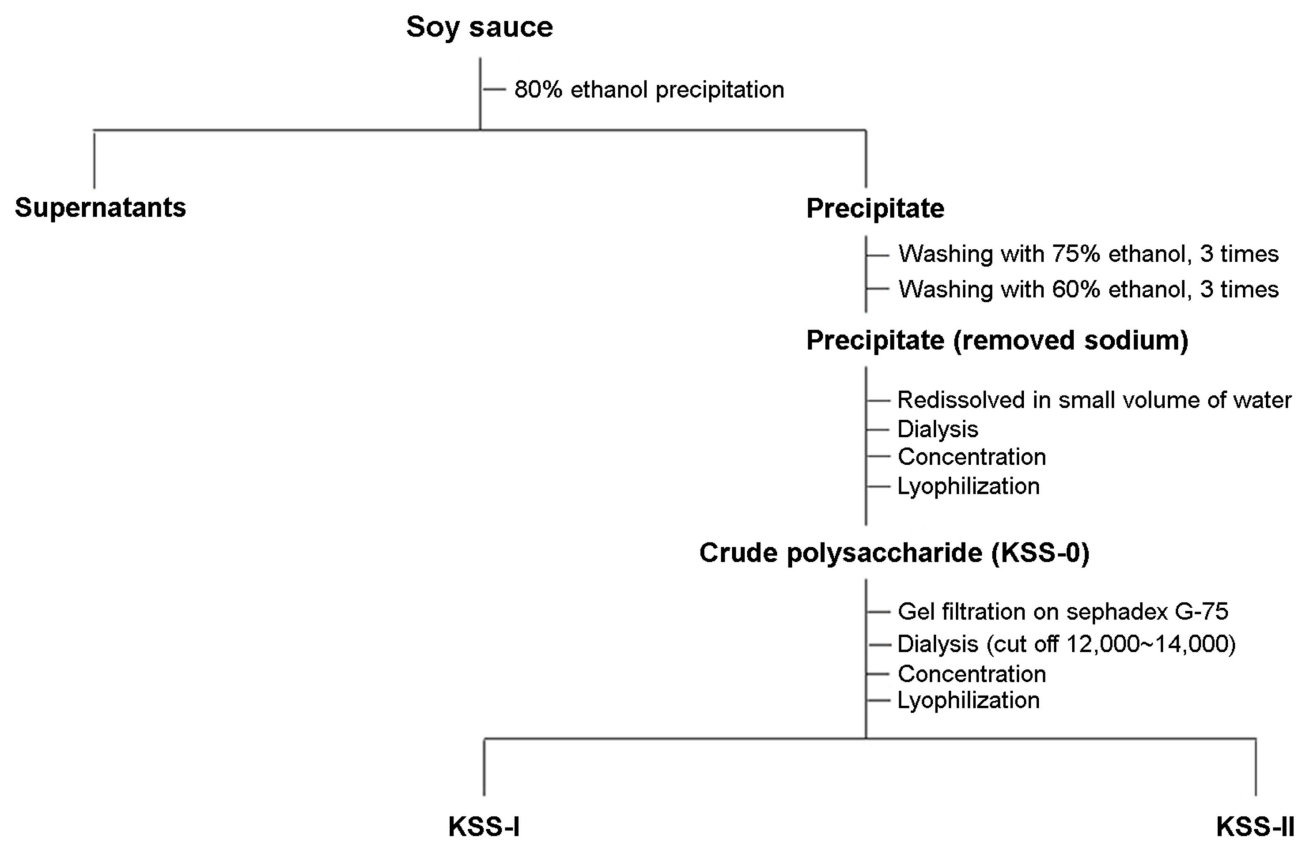

Fig. 1. Isolation and purification of crude polysaccharide isolated from Korean-style soy sauce with a Sephadex G-75 column. 
A

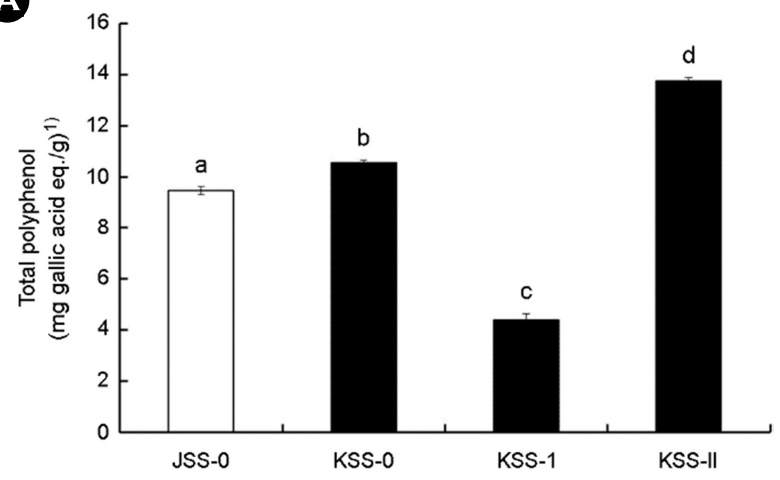

B

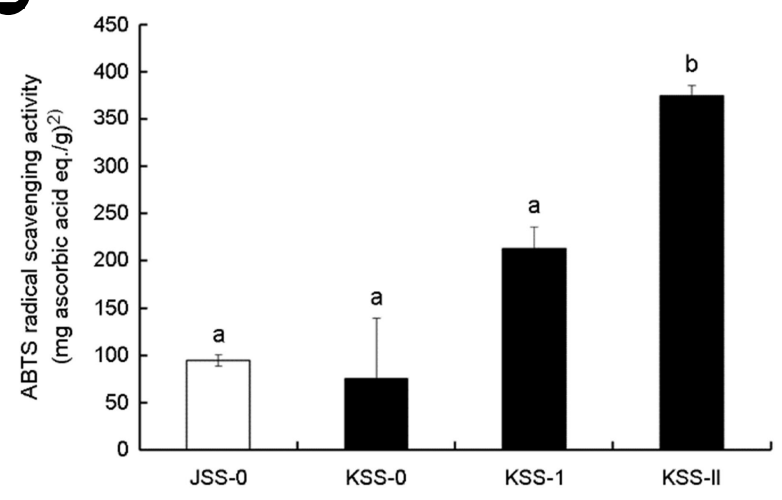

C

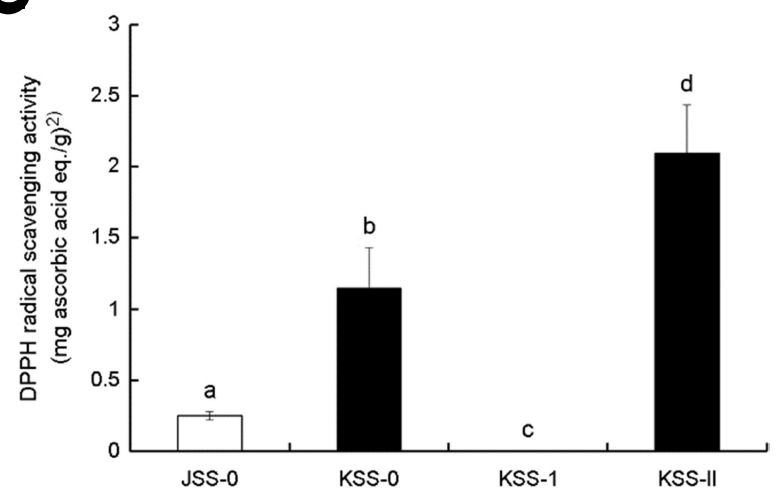

Fig. 2. Antioxidant activity of two crude polysaccharides (JSS-0 and KSS-0) and their fractions (KSS-I and KSS-II). (A) Total polyphenol contents; (B) ABTS free radical scavenging activity; (C) DPPH free radical scavenging activity. JSS-0; crude polysaccharide of Japanese soy sauce, KSS-0; crude polysaccharide of Korean soy sauce, KSS-I; fractionized polysaccharide of Korean soy sauce (high molecular weight), KSS-II; fractionized polysaccharide of Korean soy sauce (low molecular weight).

${ }^{1)} \mathrm{mg}$ gallic acid eq./g: $\mathrm{mg}$ gallic acid equivalents per gram of dry weight, ${ }^{2)} \mathrm{mg}$ ascorbic acid eq./g: $\mathrm{mg}$ ascorbic acid equivalent antioxidant capacity per gram of dry weight, ${ }^{\text {abcd }}$ Values not sharing a common superscript vary significantly $(P<0.05)$ from each other.

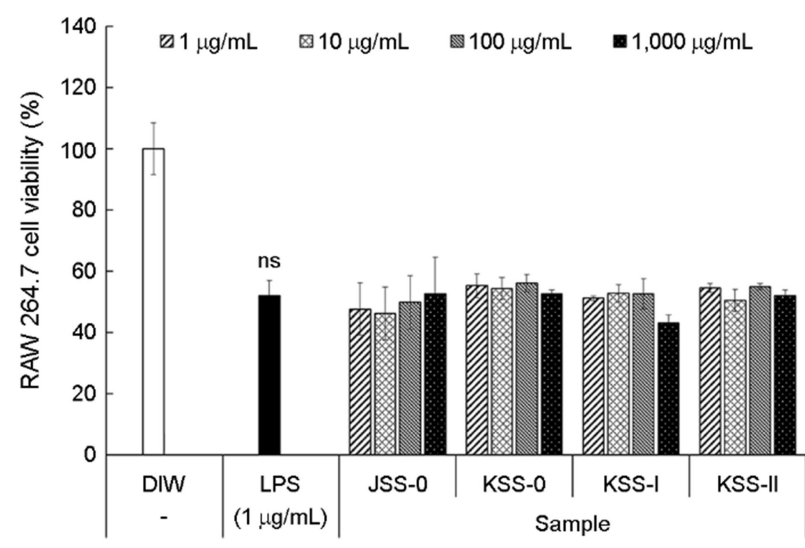

Fig. 3. Effect of two crude polysaccharides (JSS-0 and KSS-0) and their fractions (KSS-I and KSS-II) on RAW 264.7 cell viability. RAW 264.7 cells $\left(2 \times 10^{5}\right.$ cells/well) were treated with polysaccharide samples for $30 \mathrm{~min}$ followed by addition of LPS ( $1 \mu \mathrm{g} /$ $\mathrm{mL}$ ) for a further $24 \mathrm{~h}$. Viability was evaluated using the EZ-CyTox assay. JSS-0; crude polysaccharide of Japanese soy sauce, KSS-0; crude polysaccharide of Korean soy sauce, KSS-I; fractionized polysaccharide of Korean soy sauce (high molecular weight), KSS-II; fractionized polysaccharide of Korean soy sauce (low molecular weight). ns means there is not a significant difference between the LPS-treated control and respective samples by Student's $t$-test.

JSS-0, therefore, KSS-0 was fractionated into two polysaccharide fractions (KSS-I and KSS-II) using Sephadex G-75 size exclusion chromatography (Fig. 1). KSS-I had a relatively higher molecular weight, whereas KSS-II had a much lower molecular weight.

To determine anti-oxidative activity, we first measured total polyphenol content. A plant-based diet contains many phenolic compounds and they function as biologically active compounds for disease prevention (Upadhyay and Dixit, 2015). Total polyphenol content was calculated using gallic acid as a standard reagent. KSS-II contained the highest total polyphenol, $13.8 \mathrm{mg} / \mathrm{g}$ of the four polysaccharides. Also, KSS- 0 , JSS- 0 and KSS-I contained $10.5 \mathrm{mg} / \mathrm{g}, 9.5 \mathrm{mg} / \mathrm{g}$, and $4.4 \mathrm{mg} / \mathrm{g}$ of total polyphenol, respectively (Fig. 2A). KSS-II also showed the highest ABTS and DPPH radical scavenging activities. Both ABTS and DPPH radical scavenging activities were calculated using ascorbic acid as a standard reagent. KSS-II, KSS-I, JSS-0, and KSS-0 showed 374.5, 212.5, 94.4, and $75.1 \mathrm{mg} / \mathrm{g}$ ABTS radical scavenging activity, respectively (Fig. 2B). KSS-II, KSS-0, and JSS-0 showed 2.1, 1.1, and $0.2 \mathrm{mg} / \mathrm{g}$ DPPH radical scavenging 
A

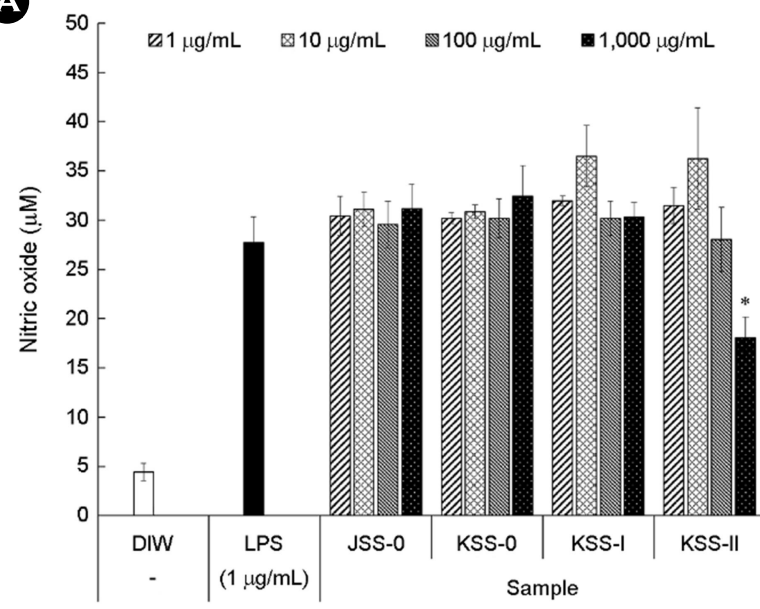

B

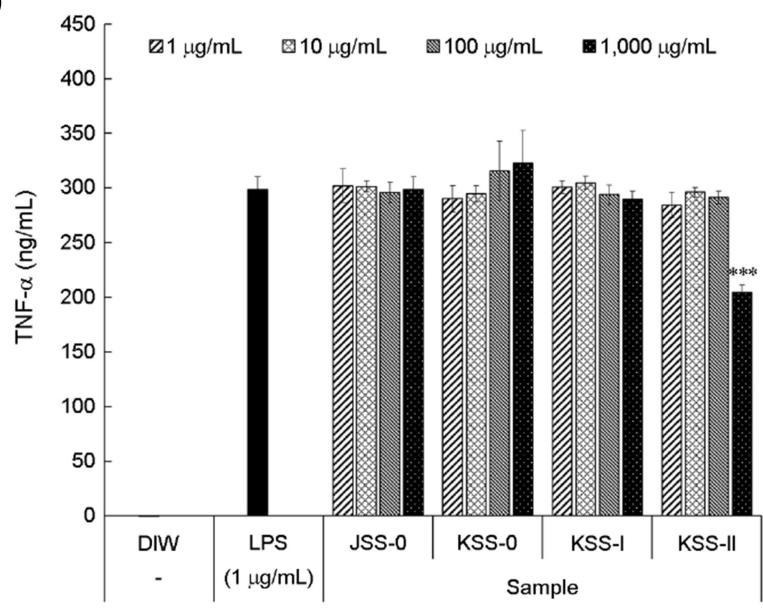

Fig. 4. Anti-inflammatory activity of two crude polysaccharides (JSS-0 and KSS-0) and their fractions (KSS-I and KSS-II) in LPS-stimulated RAW 264.7 cells. (A) NO production; (B) TNF- $\alpha$ production. RAW 264.7 cells $\left(2 \times 10^{5}\right.$ cells/well) were treated with polysaccharide samples for 30 min followed by addition of LPS $(1 \mu \mathrm{g} / \mathrm{mL})$ for a further $24 \mathrm{~h}$. Production of NO and TNF- $\alpha$ were quantified using the Griess reagent and a commercial ELISA kit, respectively. JSS-0; crude polysaccharide of Japanese soy sauce, KSS-0; crude polysaccharide of Korean soy sauce, KSS-I; fractionized polysaccharide of Korean soy sauce (high molecular weight), KSS-II; fractionized polysaccharide of Korean soy sauce (low molecular weight). Different superscripts are significantly different between the LPS-treated control and respective samples by Student's $t$-test. *, $P<0.05$; and ${ }^{* * *}, P<0.001$.

activity, respectively. KSS-I did not show DPPH radical scavenging activity (Fig. 2C).

We measured cell viability of RAW 264.7 cells to investigate the cell cytotoxicity of polysaccharides and LPS. LPS is a component of the Gram-negative cell wall and is widely used to induce inflammation (Moreillon and Majcherczyk, 2003). When RAW 264.7 cells are treated with LPS, macrophages produce inflammatory mediators such as NO, TNF- $\alpha$, interleukins, prostanoids, and leukotrienes (Lee et al., 2003). All polysaccharides did not show cell cytotoxicity up to $1,000 \mu \mathrm{g} / \mathrm{mL}$ concentration (Fig. 3).

To determine the inflammatory activity of polysaccharides, we measured two major inflammatory mediators, NO and TNF- $\alpha$. NO participates in the regulation of various immune responses and has an anti-inflammatory effect under normal physiological conditions; however, it is also a proinflammatory mediator that induces inflammation when it is produced excessively in abnormal situations (Sharma et al., 2007). TNF- $\alpha$ is a representative pro-inflammatory cytokine and has an important role in the immune system during inflammation, cell proliferation, and apoptosis (Zelová and Hošek, 2013).

As a result, only KSS-II $(1,000 \mu \mathrm{g} / \mathrm{mL})$ showed signi- ficant anti-inflammatory activity against NO and TNF- $\alpha$ production. When the LPS-treated control was set to $100 \%$, KSS-II had $34.7 \%$ and $31.4 \%$ inhibitory effect on NO and TNF- $\alpha$ production, respectively (Fig. $4 \mathrm{~A}$ and B).

In conclusion, the polysaccharide isolated from Korean soy sauce (KSS-II) showed higher anti-oxidant and antiinflammatory activities than polysaccharides isolated from Japanese soy sauce. Therefore, Korean soy sauce has better physiological activity than Japanese soy sauce, so purified polysaccharide from Korean soy sauce may be used as a beneficial material in functional foods.

\section{ACKNOWLEDGEMENT}

This work was supported by the National Research Foundation of Korea (NRF) grant funded by the Korea government (MSIT) (NRF-2017R1C1B5017634).

\section{CONFLICT OF INTEREST}

The authors have no conflicts of interest to discolose.

\section{REFERENCES}

Arnao MB, Cano A, Acosta M. The hydrophilic and lipophilic 
contribution to total antioxidant activity. Food Chemistry. 2001. 73: 239-244.

Blois MS. Antioxidant determinations by the use of a stable free radical. Nature. 1958. 181: 1199-1200.

Dore CMG, Azevedo TC, de Souza MC, Rego LA, de Dantas JC, Silva FR, Rocha HA, Baseia IG, Leite EL. Antiinflammatory, antioxidant and cytotoxic actions of $\beta$-glucan-rich extract from geastrum saccatum mushroom. International Immunopharmacology. 2007. 7: 1160-1169.

Kim HK, Cheon BS, Kim YH, Kim SY, Kim HP. Effects of naturally occurring flavonoids on nitric oxide production in the macrophage cell line raw 264.7 and their structure-activity relationships. Biochemical Pharmacology. 1999. 58: 759-765.

Kobayashi M. Immunological functions of soy sauce: Hypoallergenicity and antiallergic activity of soy sauce. Journal of Bioscience and Bioengineering. 2005. 100: 144-151.

Lee AK, Sung SH, Kim YC, Kim SG. Inhibition of lipopolysaccharide-inducible nitric oxide synthase, TNF- $\alpha$ and COX-2 expression by sauchinone effects on I- $\mathrm{kB} \alpha$ phosphorylation, C/EBP and AP-1 activation. British Journal of Pharmacology. 2003. 139: $11-20$

Moreillon P, Majcherczyk P. Proinflammatory activity of cell-wall constituents from gram-positive bacteria. Scandinavian Journal of Infectious Diseases. 2003. 35: 632-641.

Sharma J, Al-Omran A, Parvathy S. Role of nitric oxide in inflammatory diseases. Inflammopharmacology. 2007. 15: 252-259.

Shirley R, Ord EN, Work LM. Oxidative stress and the use of antioxidants in stroke. Antioxidants. 2014. 3: 472-501.

Singleton VL, Rossi JA. Colorimetry of total phenolics with phosphomolybdic-phosphotungstic acid reagents. American
Journal of Enology and Viticulture. 1965. 16: 144-158.

Tak PP, Firestein GS. NF-kB: A key role in inflammatory diseases. The Journal of Clinical Investigation. 2001. 107: 7-11.

Tsuchiya H, Sato M, Watanabe I. Antiplatelet activity of soy sauce as functional seasoning. Journal of Agricultural and Food Chemistry. 1999. 47: 4167-4174.

Uchida M, Hideshima N, Araki T. Development of koji by culturing aspergillus oryzae on nori (pyropia yezoensis). Journal of Bioscience and Bioengineering. 2019. 127: 183-189.

Upadhyay S, Dixit M. Role of polyphenols and other phytochemicals on molecular signaling. Oxidative Medicine and Cellular Longevity. 2015. 2015: ID 504253.

Wang H, Liu Y, Qi Z, Wang S, Liu S, Li X, Wang H, Xia X. An overview on natural polysaccharides with antioxidant properties. Current Medicinal Chemistry. 2013. 20: 2899-2913.

Yang HJ, Kwon DY, Kim MJ, Kang S, Park S. Meju, unsalted soybeans fermented with bacillus subtilis and aspergilus oryzae, potentiates insulinotropic actions and improves hepatic insulin sensitivity in diabetic rats. Nutrition \& Metabolism. 2012. 9: 37.

Zelová H, Hošek J. TNF- $\alpha$ signalling and inflammation: Interactions between old acquaintances. Inflammation Research. 2013. 62: 641-651.

https://doi.org/10.15616/BSL.2020.26.1.51

Cite this article as: Kim H, Park J, Jung J, Hwang D. Anti-oxidative and Anti-inflammatory Activities of Polysaccharide isolated from Korean-Style Soy Sauce. Biomedical Science Letters. 2020. 26: 51-56. 